\title{
The Expression of PHOSPHO1, nSMase 2 and TNAP is Coordinately Regulated by Continuous PTH Exposure in Mineralising Osteoblast Cultures
}

\author{
D. A. Houston ${ }^{1} \cdot$ K. Myers ${ }^{1}$ - V. E. MacRae ${ }^{1}$ - K. A. Staines ${ }^{1}$ - C. Farquharson'
}

Received: 22 March 2016/Accepted: 12 July 2016/Published online: 21 July 2016

(c) The Author(s) 2016. This article is published with open access at Springerlink.com

\begin{abstract}
Sustained exposure to high levels of parathyroid hormone (PTH), as observed in hyperparathyroidism, is catabolic to bone. The increase in the RANKL/OPG ratio in response to continuous $\mathrm{PTH}$, resulting in increased osteoclastogenesis, is well established. However, the effects of prolonged PTH exposure on key regulators of skeletal mineralisation have yet to be investigated. This study sought to examine the temporal expression of PHOSPHO1, TNAP and nSMase2 in mineralising osteoblast-like cell cultures and to investigate the effects of continuous PTH exposure on the expression of these enzymes in vitro. PHOSPHO1, nSMase2 and TNAP expression in cultured MC3T3-C14 cells significantly increased from day 0 to day 10 . PTH induced a rapid downregulation of Phosphol and Smpd3 gene expression in MC3T3-C14 cells and cultured hemi-calvariae. Alpl was differentially regulated by PTH, displaying upregulation in cultured MC3T3-C14 cells and downregulation in hemicalvariae. PTH was also able to abolish the stimulatory effects of bone morphogenic protein 2 (BMP-2) on Smpd3 and Phosphol expression. The effects of PTH on Phosphol expression were mimicked with the cAMP agonist forskolin and blocked by the PKA inhibitor PKI (5-24), highlighting a role for the cAMP/PKA pathway in this regulation. The potent down-regulation of Phosphol and Smpd3 in osteoblasts in response to continuous PTH may provide a novel explanation for the catabolic effects on the skeleton of such an exposure. Furthermore, our findings support the hypothesis that PHOSPHO1, nSMase2 and
\end{abstract}

D. A. Houston

dean.houston@roslin.ed.ac.uk

1 The Roslin Institute and R(D)SVS, University of Edinburgh, Easter Bush, Midlothian EH25 9RG, Scotland, UK
TNAP function cooperatively in the initiation of skeletal mineralisation.

Keywords Parathyroid hormone - Osteoblast · Mineralisation regulators · Hyperparathyroidism · PHOSPHO1

\section{Introduction}

Phosphatases are essential regulators of skeletal mineralisation, modifying local levels of inorganic phosphate (Pi), inorganic pyrophosphate (PPi) and the phosphorylation status of osteopontin, a key regulator of extracellular matrix (ECM) mineralisation [1]. The importance of tissuenonspecific alkaline phosphatase (TNAP) as an essential component of successful skeletal mineralisation is perhaps most striking in the condition known as hypophosphatasia (HPP). Individuals possessing hypomorphic ALPL (the gene encoding TNAP in humans) mutations typically present with severe rickets and osteomalacia resulting in a blockade of hydroxyapatite propagation within the ECM [2]. Despite this, chondrocyte- and osteoblast-derived matrix vesicles (MV) from both HPP patients and $\mathrm{Alpl}^{-1-}$ mice retain the ability to initiate intra-vesicular mineralisation and contain hydroxyapatite crystals [3, 4], highlighting that TNAP is not essential for the initiation of MVmediated ECM mineralisation.

Active within chondrocyte- and osteoblast-derived MV $[5,6]$ and expressed exclusively in mineralising bone, cartilage and dentin [7-9], PHOSPHO1, a member of the haloacid dehalogenase superfamily, is essential for the initiation of MV-mediated mineralisation. Small-molecule inhibition of PHOSPHO1 in MV's derived from $\mathrm{Alpl}^{-1-}$ mice significantly affects their ability to calcify in vitro [7]. 
The genetic ablation of Phosphol results in severely hypomineralised skeletal and dental tissues with resulting bowing of the long bones, spontaneous fractures and scoliosis [9-12]. Complete ablation of skeletal mineralisation is observed in Phosphol ${ }^{-1-} ; \mathrm{Alpl}^{-1-}$ double-knockout embryos and in metatarsals cultured in the presence of both PHOSPHO1 and TNAP inhibitors $[11,13]$. In vitro, PHOSPHO1 shows high phosphohydrolase activity towards phosphocholine and phosphoethanolamine, two metabolites derived from lipid metabolism [5]. More recently, hypomineralisation of skeletal and dental tissues was observed in the fro/fro mouse, a mouse containing a major deletion in the sphingomyelinase phosphodiesterase 3 (Smpd3) gene [14, 15]. Smpd3 encodes for neutral sphingomyelinase 2 (nSMase2), which catalyses the breakdown of the membrane lipid sphingomyelin to ceramide and phosphocholine. Through their role in the generation and processing of phosphocholine, nSMase 2 and PHOS$\mathrm{PHO1}$, respectively, may function together to liberate $\mathrm{Pi}$ for skeletal mineralisation. In spite of our knowledge of the skeletal pathophysiology in the absence of Phosphol, little is known about the regulation of Phosphol expression.

A recent RNA-seq analysis by St John et al. [16] revealed that Phosphol and Smpd3 expression are differentially regulated by parathyroid hormone (PTH) treatment of IDG-SW3 cells (an osteocyte cell line). This study extends a growing body of evidence revealing that PTH can regulate a plethora of genes involved in osteoblast function [17]. Indeed, a microarray study of PTH-treated UMR-106-01 cells, a rat osteosarcoma cell line, revealed $>100$ differentially regulated genes, with the gene profile closely mimicking the gene expression pattern observed during osteoblast differentiation [18]. The transcription and posttranslational modification (phosphorylation) of Cbfa1, the transcription factor and master regulator of osteoblast differentiation are also strongly enhanced by PTH [19]. Alterations in osteoblast gene expression by PTH may underscore PTH ability to elicit both anabolic and catabolic effects on the skeleton depending on the duration of the exposure. For example, daily injections of low-dose PTH are anabolic to the skeleton, whereas continuous exposure to PTH, the likes observed in hyperparathyroidism, results in bone loss and increased porosity especially in the cortical compartment [20]. Indeed, despite an overall increase in bone remodelling [21], the enhancement of receptor activator of nuclear factor- $\kappa \mathrm{B}$ ligand (RANKL) expression and thus osteoclastogenesis, and the suppression of the RANKL decoy receptor, osteoprotegerin [22], ensure that any increases in bone formation are outweighed by a prevailing bone resorption response. Furthermore, the bone formation response that occurs is predominated by osteoid production rather than a true mineralised bone matrix [23]. The reduction in bone mineral density associated with hyperparathyroidism [24] may be a direct effect of PTH on key regulators of mineralisation. This study, therefore, sought to examine the effect of a short-term continuous PTH exposure on PHOSPHO1, TNAP and nSMase2 expression in osteoblast-like cells and calvariae explants as well as to examine the PTH signal transduction pathways involved.

\section{Results}

\section{Temporal Gene and Protein Expression and Matrix Mineralisation in MC3T3 Cells}

MC3T3-C14 cells cultured in the presence of ascorbic acid and calcium chloride mineralised their ECM by day 10 . Quantification of leached Alizarin red confirmed a significant increase in mineral content on day 10 compared with day 7 of culture $(0.775 \pm 0.145$ vs. $0.125 \pm 0.01$ relative absorbance units, $P<0.001$; Fig. 1a, b). Phosphol mRNA expression markedly increased in a temporal manner and by day 10 in culture mRNA levels were $\sim 150$-fold higher $(P<0.001)$ compared to day 0 (Fig. $1 \mathrm{c})$. A similar tem-

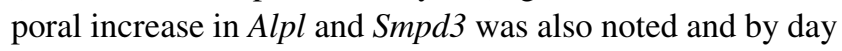
10 in culture where the expression of both was increased by $>60$-fold $(P<0.001)$ compared to day 0 (Fig. 1d, e). The changes in gene expression were similarly observed at the protein level by western blotting (Fig. 1f). The expression of both PHOSPHO1 protein bands, which are considered to be a result of alternative start sites, was increased similarly with time in culture [8].

\section{The Effects of Continuous PTH on Matrix Mineralisation in MC3T3 Cells}

The addition of $50 \mathrm{nM}$ PTH every $48 \mathrm{~h}$ to MC3T3-C14 cell cultures for 10 days caused a reduction in the extent of matrix mineralisation compared to control cultures as assessed by the quantitative alizarin red assay $(P<0.05$; Fig. 1g).

\section{The Effects of Continuous PTH on Gene and Protein Expression in MC3T3 Cells}

Initial experiments assessed the ability of varying concentrations of PTH to regulate Phosphol, Alpl and Smpd3 expression by MC3T3-C14 cells. MC3T3-C14 cells were treated with $0.05-50 \mathrm{nM}$ PTH for $24 \mathrm{~h}$ on day 6 of culture, when initial mineral formation could be observed with light microscopy. At concentrations of $0.5 \mathrm{nM}$ and above, PTH significantly down-regulated Phosphol mRNA expression $(P<0.001$; Fig. 2a). PTH increased $A l p l$ expression at the two highest concentrations examined, $25 \mathrm{nM}(P<0.05)$ 
Fig. 1 Extracellular matrix mineralisation and temporal gene expression in MC3T3-C14 cells. a Alizarin red staining and b relative quantification of cetylpyridinium chloride leached bound dye. RT-qPCR analysis of c Phosphol d Alpl and e Smpd3 mRNA expression in mineralising MC3T3-C14 cells over a 10-day culture period. f Western blotting analysis of PHOSPHO1, TNAP and nSMase2 in MC3T3-C14 cells over a 10-day culture period. The fold change in fluorescence intensity against cultures at day 0 (normalised against beta-actin) is shown below each protein of interest. g Assessment of matrix mineralisation in MC3T3-C14 cell cultures continuously exposed to PTH for 10 days. Quantification of, cetylpyridinium chloride leached, bound Alizarin red staining. $N=3 ; * P<0.05$, $* * P<0.01 ; * * * P<0.001$ in comparison with the previous time point in culture
A

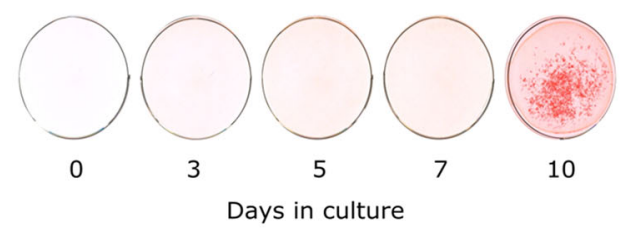

C

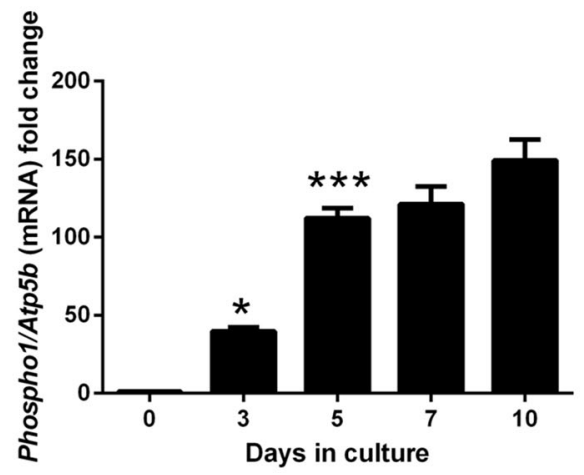

E

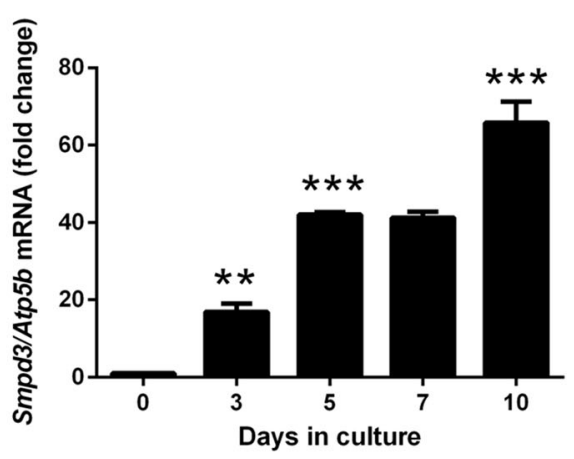

G

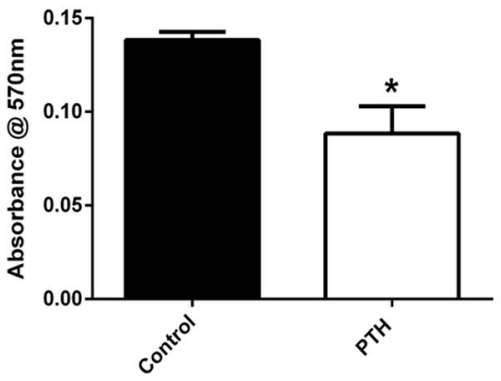

B

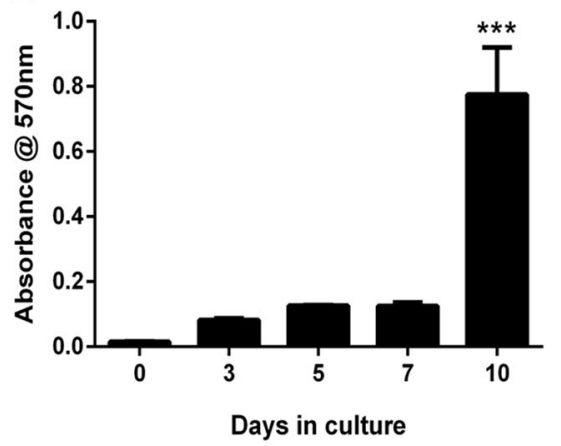

D

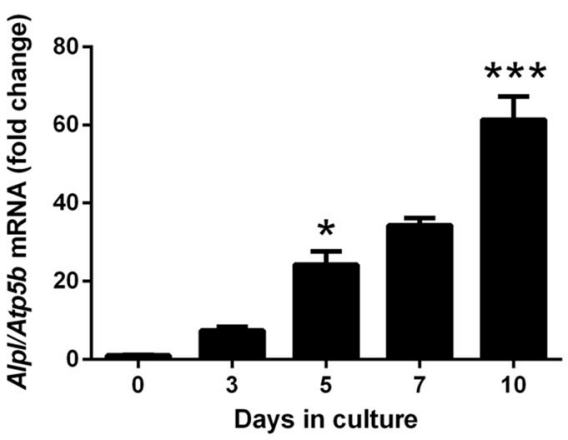

Days in culture

$\mathbf{F}$

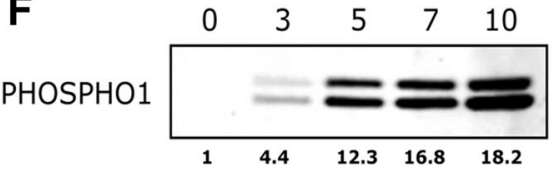

TNAP

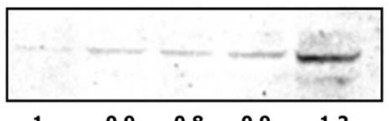

nSMase2

$\begin{array}{lllll}1 & 0.9 & 0.8 & 0.9 & 1.2\end{array}$

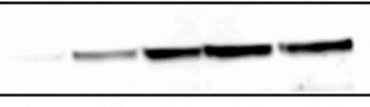

$\beta$-Actin

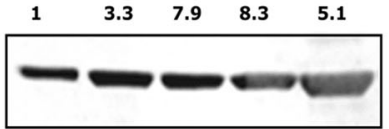



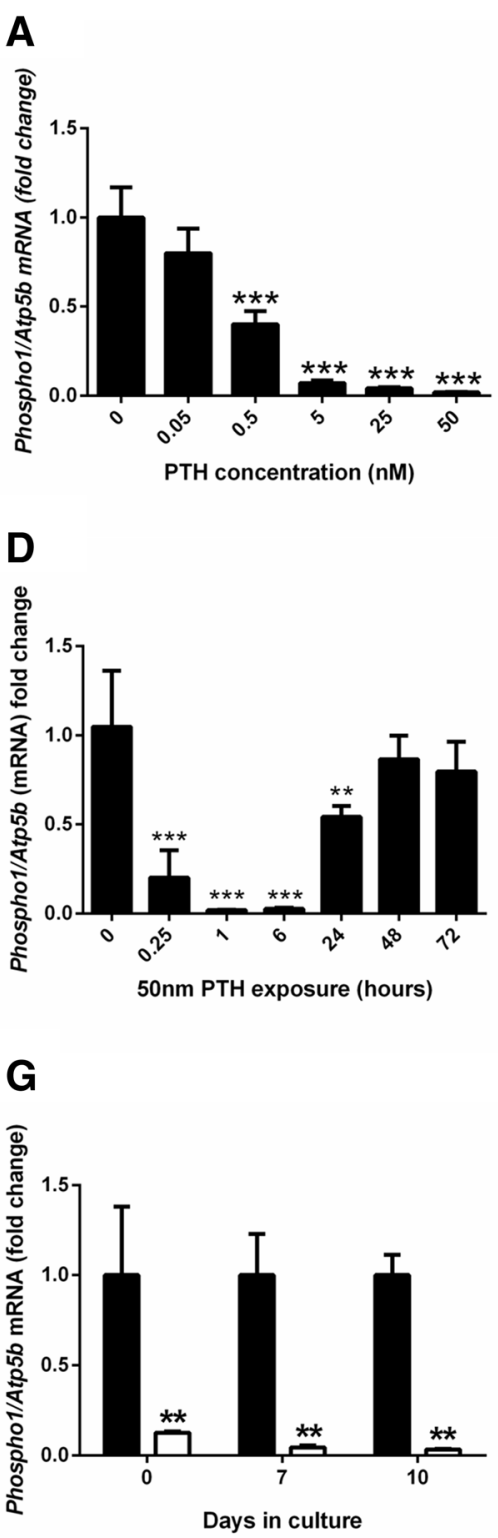

J

bPTH (1-34) concentration (nM)

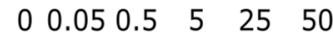
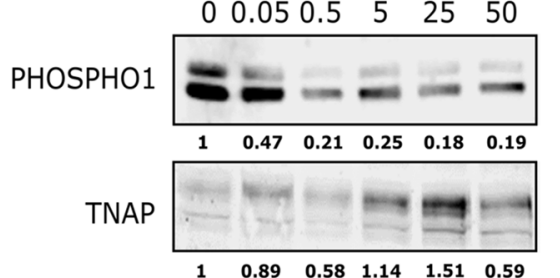

nSMase2

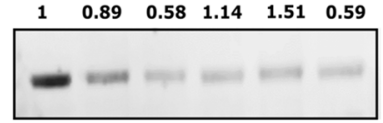

$\beta$-Actin
B

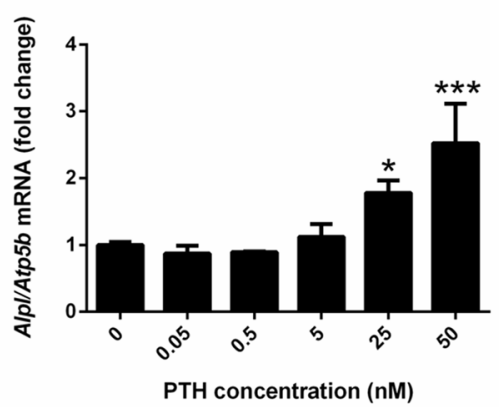

E

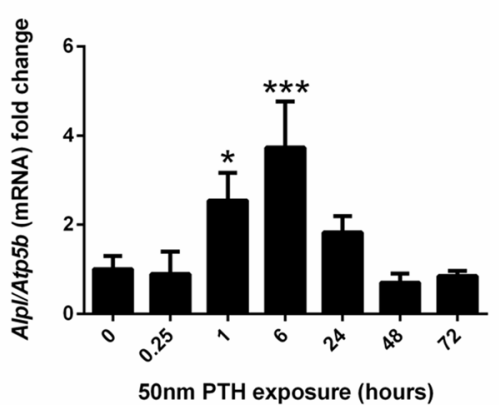

H

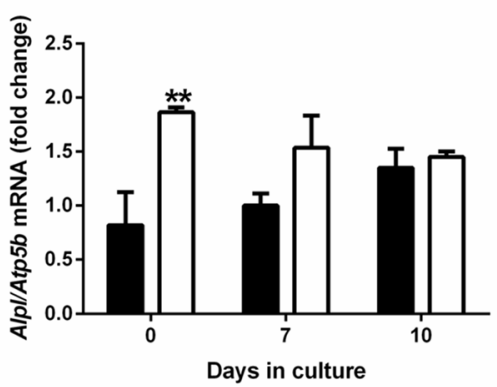

K

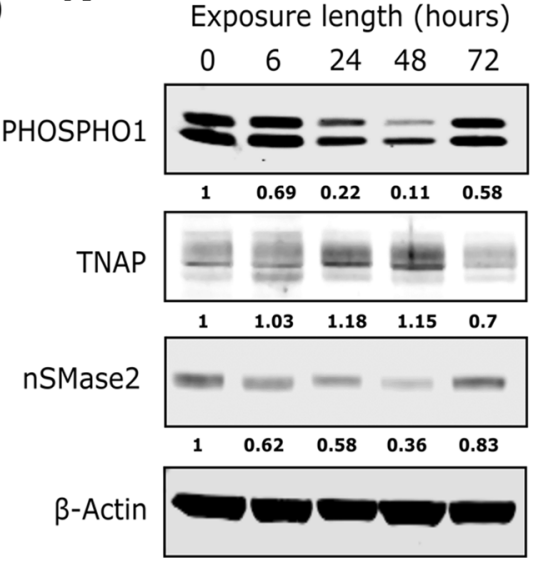

C

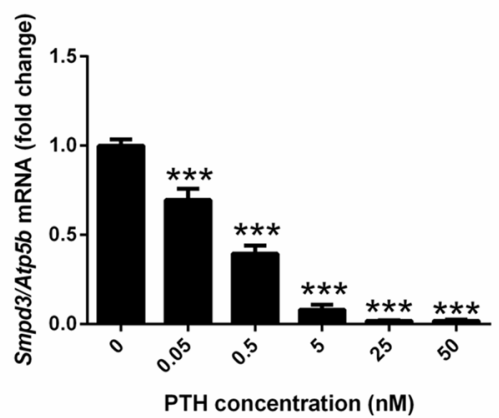

$\mathbf{F}$

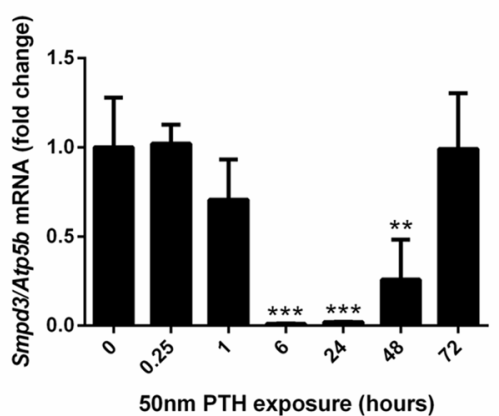

I

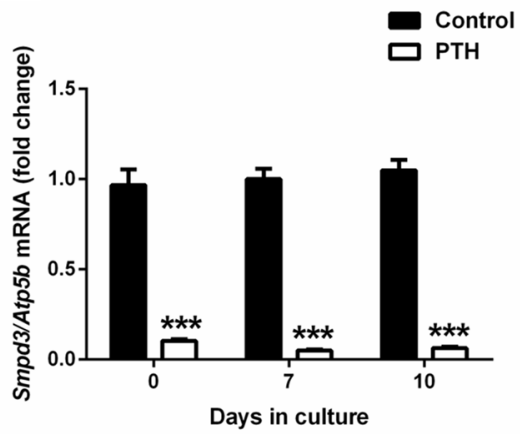

Days in culture

$\mathbf{L}$
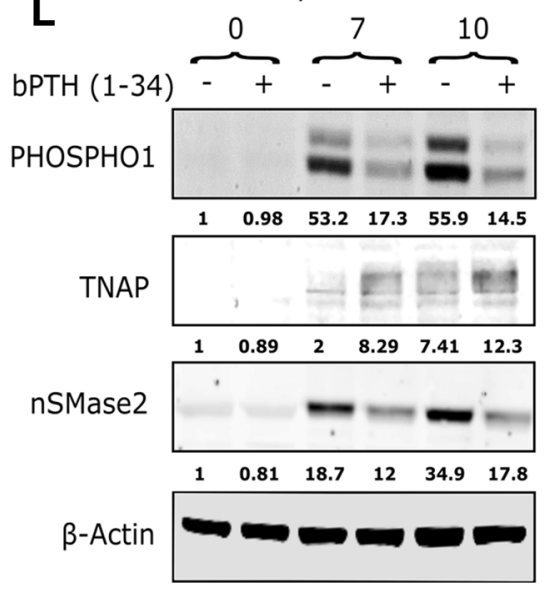
४Fig. 2 Regulation of key mineralisation genes by PTH in MC3T3C14 cells. RT-qPCR analysis of a Phospho1, b Alpl and c Smpd3 mRNA expression in response to a 24-h exposure of various doses of bPTH (1-34) on day 6 of culture. RT-qPCR analysis of $\mathbf{d}$ Phosphol, e $A l p l$ and $\mathbf{f} S m p d 3$ mRNA expression in response to various exposure times of bPTH (1-34) (50 nM). The timing of PTH addition was adjusted to ensure all experiments finished on day 7 of culture. RTqPCR analysis of $\mathbf{g}$ Phosphol, $\mathbf{h}$ Alpl and $\mathbf{i}$ Smpd3 mRNA expression in response to 24-h exposure PTH $(50 \mathrm{nM})$ in MC3T3-C14 cells at different stages of differentiation (confluency (day 0), days 7 and day 10 post-confluency). j Western blotting analysis of PHOSPHO1, TNAP, nSMase2 in response to a 24-h exposure of various doses of PTH. The fold change in fluorescence intensity against control treated cultures (normalised against beta-actin) is shown below each protein of interest. k Western blotting analysis of PHOSPHO1, TNAP, $\mathrm{nSmase} 2$ in response to various exposure times of PTH $(50 \mathrm{nM})$. The fold change in fluorescence intensity against control treated cultures (normalised against beta-actin) is shown below each protein of interest. I Western blotting analysis of PHOSPHO1, TNAP, nSMase2 in response a 24-h exposure PTH $(50 \mathrm{nM})$ in MC3T3-C14 cells at different stages of differentiation. The fold change in fluorescence intensity against day 0 control treated cultures (normalised against beta-actin) is shown below each protein of interest. $N=3$; $* P<0.05 ; * * P<0.01 ; * * * P<0.001$ in comparison with controls

and $50 \mathrm{nM}(P<0.001)$, whereas it down-regulated Smpd3 expression at all concentrations examined $(P<0.001$; Fig. 2b, c). Western blotting confirmed that the changes in gene expression were replicated at the protein level (Fig. 2j).

We next examined the temporal effects of PTH on Phospho1, Alpl and Smpd3 expression at day 7 of culture (matching the end point in experiments described in Fig. 2a-c). PTH rapidly down-regulated Phosphol after 15 min which was further exacerbated after 1 -h and 6-h exposures $(P<0.001$; Fig. 2 d). By 24 h, the down-regulation of Phosphol by PTH was lessened $(P<0.05)$, and after 48-h and 72-h exposures Phosphol expression had returned to basal levels (Fig. 2d). Enhancement of Alpl expression by PTH was noted after $1 \mathrm{~h}(P<0.05)$, peaking at $6 \mathrm{~h}(P<0.001)$ before returning to control levels after 24-h and longer exposures (Fig. 2e). The expression pattern of Smpd3 in response to PTH was similar to that of Phosphol; however, the speed of reduced expression and its normalisation was slower (Fig. 2f). Changes in protein levels of PHOSPHO1, TNAP and nSMase2 reflected their respective changes in gene expression (Fig. 2k). Notably, the expression levels of PHOSPHO1 and nSMase 2 were less, and TNAP was greater after 24-h and 48-h PTH exposure (Fig. 2k). After a 72-h exposure all proteins were expressed at a comparable level to the control treated cultures.

Finally, the effect of PTH $(50 \mathrm{nM}, 24 \mathrm{~h})$ on the expression of Phosphol, Alpl and Smpd3 was assessed in MC3T3-C14 cells at different stages of differentiation. Phosphol and Smpd3 regulation by PTH was not dependent on the differentiation status of the cell cultures, with both genes significantly down-regulated $(P<0.001)$ at 0,7 and 10 days in culture (Fig. $2 \mathrm{~g}$, i). In contrast, PTH-induced expression of $A l p l$ was more restricted and only reached significance at day $0(P<0.01$; Fig. 2 h). Little or no PHOSPHO1, nSMase 2 or TNAP protein was expressed at day 0 in culture, and this was not altered by PTH treatment (Fig. 21). At days 7 and 10, however, PTH decreased PHOSPHO1 and nSMase 2 expression whilst increasing TNAP expression, consistent with the gene expression data (Fig. 21).

\section{The Effects of Continuous PTH on Gene Expression in Murine Calvariae}

Murine hemi-calvariae were treated with $50 \mathrm{nM}$ PTH for 2, 6 and 24 h. A significant downregulation of both Phosphol and Smpd3 expression in response to PTH was observed at $2 \mathrm{~h}(P<0.05)$, 6 and $24 \mathrm{~h}(P<0.001$; Fig. 3a, c). Likewise, continuous PTH exposure induced a down-regulation of Alpl expression, albeit only with 24-h exposure $(P<0.01$; Fig. 3b).

\section{The Effects of Cycloheximide on PTH on Phospho1, Alpl and Smpd3 Gene Regulation}

To determine whether PTH directly regulates Phosphol, Alpl and Smpd3 gene expression, MC3T3-C14 cells were pre-treated for $1 \mathrm{~h}$ with the protein synthesis inhibitor cycloheximide $(25 \mu \mathrm{M})$, followed by treatment with $50 \mathrm{nM}$ PTH for $6 \mathrm{~h}$. Cycloheximide treatment alone did not affect basal levels of Phosphol, Alpl or Smpd3. In the presence of cycloheximide, PTH reduced the expression of Phosphol (44.1\%,P<0.01), but this was not to the same extent observed in the absence of cycloheximide (93.9\%, $P<0.001 ;$ Fig. 4a). Cycloheximide treatment did not affect the potent regulation of Alpl and Smpd3 expression by PTH (Fig. 4b, c).

\section{Identification of the Intracellular Signalling Pathways Responsible for PTH Regulation of Phospho1, Alpl and Smpd3 Expression}

Binding of PTH to the G-protein-coupled receptor PTHR1 primarily activates cAMP/PKA and PKC signalling pathways [25]. To determine which of these pathways was utilised by PTH to regulate Phosphol, Alpl and Smpd3 expression, we treated MC3T3-C14 cells and murine hemicalvariae with the cAMP inducer forskolin $(10 \mu \mathrm{M})$ or the PKC activator, PMA (100 nM) for $24 \mathrm{~h}$. In MC3T3-C14 cells, forskolin replicated the effects of $50 \mathrm{nM}$ PTH by inducing significant decreases in Phosphol $(P<0.001)$ and Smpd3 expression $(P<0.001)$ whilst significantly increasing the expression of $A l p l(P<0.001)$ PMA treated 

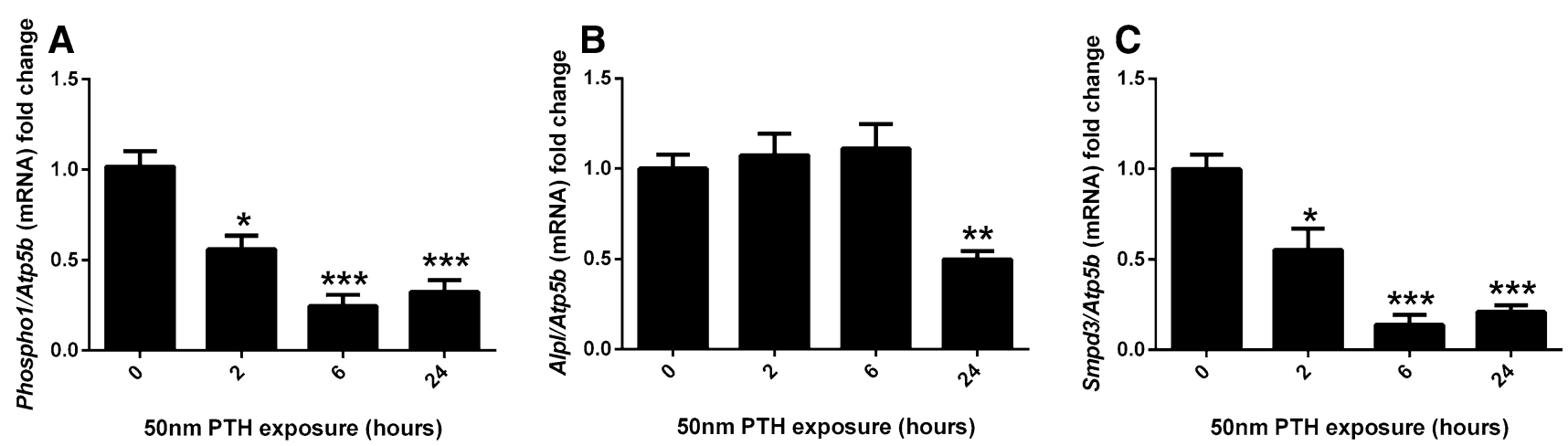

Fig. 3 Regulation of key mineralisation genes by PTH in cultured calvariae. RT-qPCR analysis of a Phosphol, b Alpl and c Smpd3 mRNA expression in response to various exposure times of PTH $(50 \mathrm{nM}) . N>3$ per time point; $* P<0.05$; *** $P<0.001$ in comparison with control

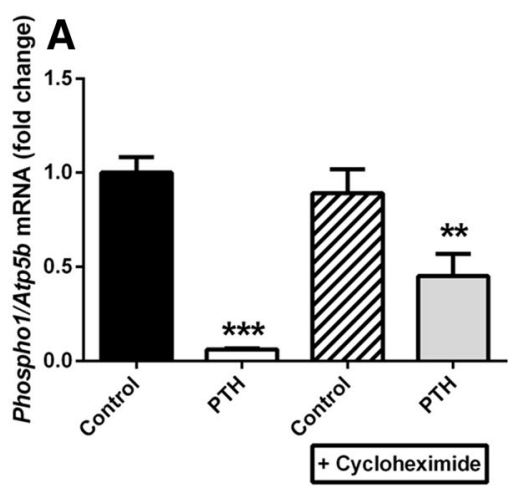

Fig. 4 Effects of bPTH (1-34) on Phospho1, Alpl and Smpd3 in MC3T3-C14 cells does not require protein synthesis. MC3T3-C14 cells were treated for $6 \mathrm{~h}$ with $50 \mathrm{nM}$ PTH or vehicle control after a 2-h pre-treatment with $50 \mu \mathrm{M}$ cycloheximide (CHX) or solvent

cells displayed no differences in Phosphol, Alpl or Smpd3 expression compared with control cultures (Fig. 5a-c). The gene expression results in MC3T3-C14 cells were confirmed at the protein level by western blotting (Fig. 5d). In hemi-calvariae, forskolin similarly replicated the effects of $50 \mathrm{nM}$ PTH by inducing a significant decrease in Phosphol $(P<0.05)$ Smpd3 $(P<0.001)$ Alpl $(P<0.001)$ expression (Fig. 5e-g). PMA-treated hemi-calvariae displayed no differences in Phosphol expression; however, the expression of $A l p l$ and Smpd3 was strongly down-regulated by exposure to PMA $(P<0.001)$. To confirm the role of the PKA signalling pathway as the mediator of the effects of PTH in MC3T3-C14 cells, cell cultures were treated with the PKA inhibitor PKI (5-24) $(100 \mathrm{nM})$ alone or in the presence of PTH for $6 \mathrm{~h}$. The addition of PKI (5-24) alone did not alter the basal expression of Phosphol, Alpl or Smpd3. The addition of the PKA inhibitor reduced the ability of $50 \mathrm{nM}$ PTH to significantly inhibit the expression of Phosphol and Smpd3 (Fig. 6a, c). Similarly, the induction of Alpl expression by PTH was abrogated by the co-incubation of PKI (5-24) (Fig. 6b). In cultured hemi-

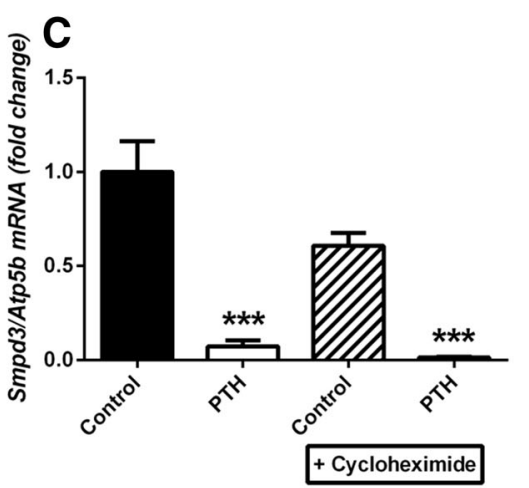

control on day 7 in culture. a Phospho1, b Alpl and c Smpd3 mRNA expression was assessed by RT-qPCR. $\quad N=3 ; \quad * P<0.05$; $* * P<0.01 ; * * * P<0.001$ in comparison with vehicle control

calvariae, PKI (5-24) inhibited the down-regulation of Phosphol by PTH, whereas the down-regulation of Smpd3 by PTH was not affected by PKI $(5-24) \quad(P<0.001$; Fig. 6f).

\section{Assessment of Transcription Factor Expression in Response to PTH in Cultured MC3T3 Cells and Murine Calvariae}

The expression of the transcription factors Runx2, Sp7, Atf4 and Trps 1 was assessed by RT-qPCR in MC3T3 cell cultures and cultured murine calvariae in response to a shortterm PTH exposure. In MC3T3 cells, the expression of Runx 2 and Trps1 did not change in response to PTH exposure (Fig. 7a, d). $S p 7$ expression was reduced after a 6-h PTH exposure $(P<0.01$; Fig. $7 b)$, whereas Atf4 was enhanced after 15 -min PTH exposure $(P<0.01$; Fig. $7 \mathrm{c})$. In cultured murine calvariae, the expression of Runx2, Atf4 and Trpsl was not altered in response to PTH exposure (Fig. 7e, g, h). Sp7 expression was significantly downregulated after a 2-h PTH exposure $(P<0.05$; Fig. 7f). 

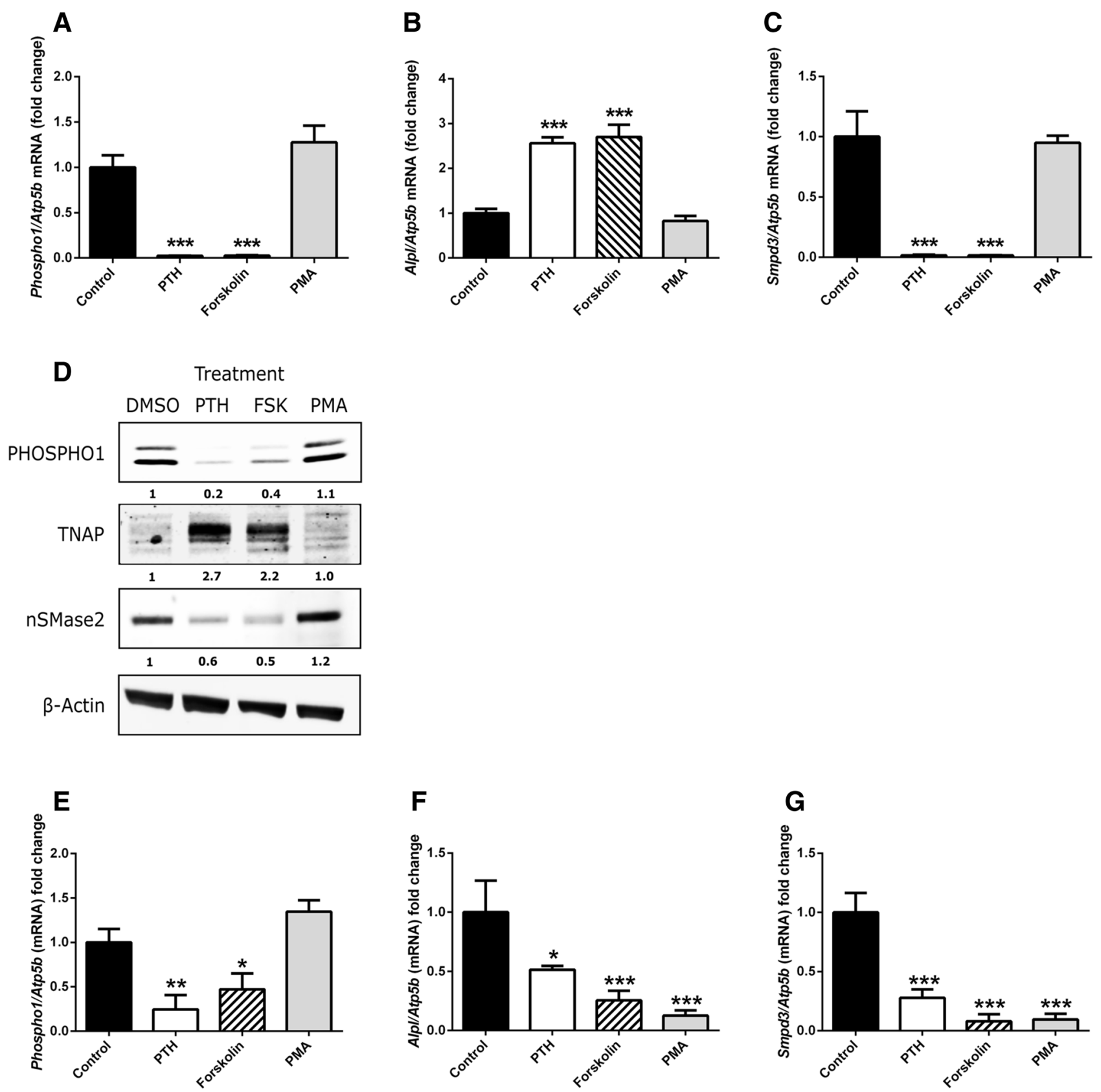

Fig. 5 Investigation of signalling pathways regulating the effects of PTH. MC3T3-C14 cells were treated with $50 \mathrm{nM} \mathrm{PTH,} 25 \mu \mathrm{M}$ forskolin, $100 \mathrm{nM}$ PMA or vehicle control for $24 \mathrm{~h}$ on day 6 in culture. a Phospho1, b Alpl and c Smpd3 mRNA expression was assessed by RT-qPCR. d Western Blotting analysis of MC3T3-C14

\section{Investigating the Combined Effects of Continuous PTH and BMP-2 Exposure on Gene Expression in MC3T3 Cells}

To investigate the effects of continuous PTH exposure in MC3T3 cells treated with BMP-2, MC3T3 cell cultures were cultured with either $50 \mathrm{nM}$ PTH, $300 \mathrm{ng} / \mathrm{mL}$ BMP-2 cells treated as described above. Hemi-calvariae were treated with $50 \mathrm{nM}$ PTH, $25 \mu \mathrm{M}$ forskolin, $100 \mathrm{nM}$ PMA or vehicle control for 24 h. e Phosphol, f Alpl and g Smpd3 mRNA expression was assessed by RT-qPCR. $N=3$; $* P<0.05$; ** $P<0.01$; *** $P<0.001$ in comparison with vehicle control

or both for $24 \mathrm{~h}$ on day 6 of culture, and the expression of Phosphol, Alpl and Smpd3 was assessed by RT-qPCR. BMP-2 exposure significantly induced the expression of Phospho1, Alpl and Smpd3 ( $P<0.001$; Fig. 8a-c). The stimulatory effect of BMP-2 on Phosphol expression was nullified by the dual exposure of PTH which caused Phosphol expression to return to unstimulated control 

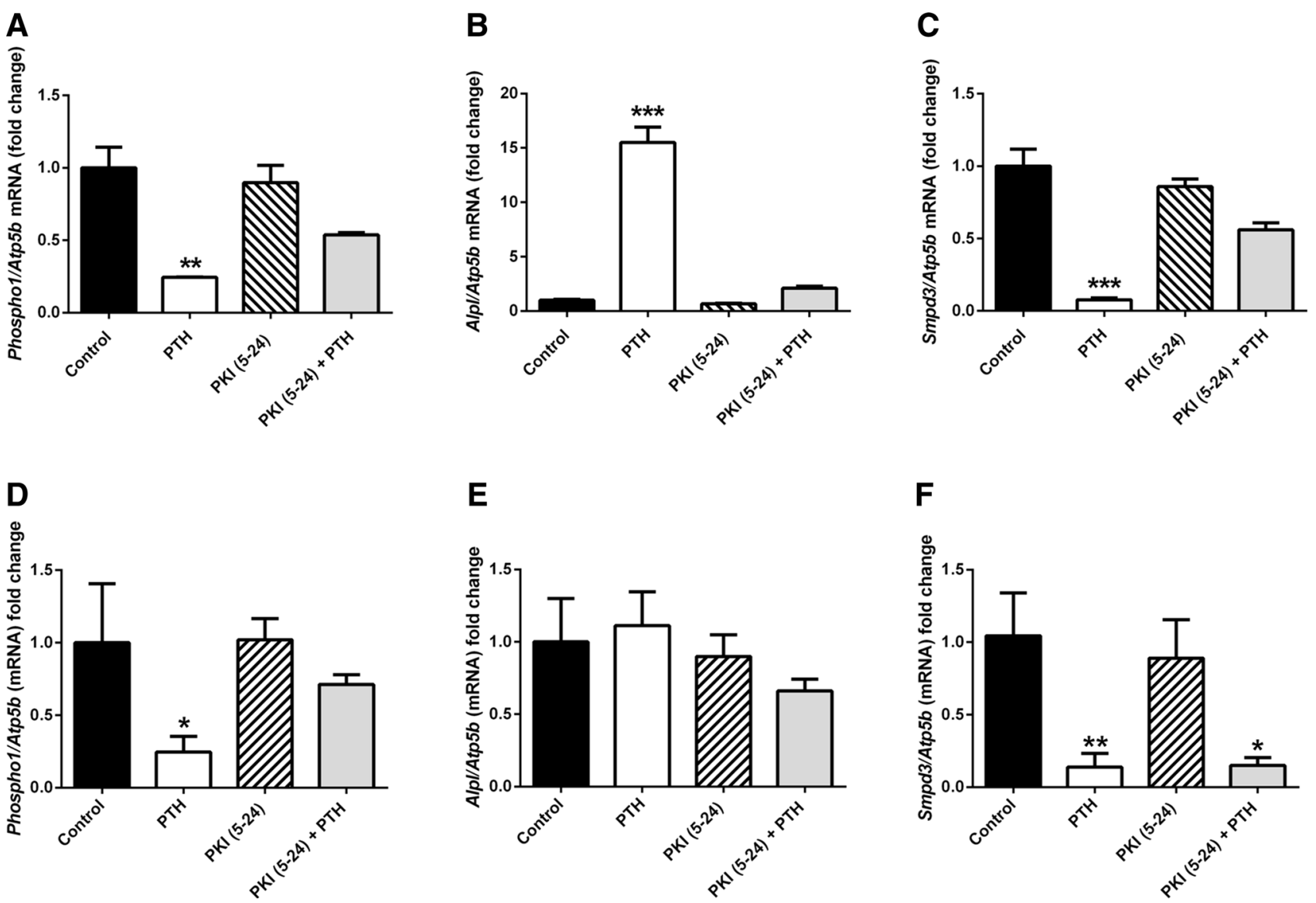

Fig. 6 Inhibition of PKA signalling pathway abolishes the effects of PTH (1-34). MC3T3-C14 cells and hemi-calvariae were treated with $50 \mathrm{nM}$ PTH or vehicle control for $6 \mathrm{~h}$ following a 1-h pre-treatment with 100 nM PKI (5-24) on day 6 of culture. a Phospho1, b Alpl and

c Smpd3 mRNA expression in MC3T3-C14 and d Phosphol, e Alpl and $\mathbf{f} \operatorname{Smpd} 3 \mathrm{mRNA}$ expression in hemi-calvariae, all assessed by RTqPCR. $N=3 ; * P<0.05 ; * * P<0.01, * * * P<0.001$ in comparison with vehicle control

levels (Fig. 8a). In contrast, the Smpd3 expression in cells treated with PTH or PTH and BMP-2 was similar, suggesting that Smpd3 expression was highly sensitive to PTH exposure $(P<0.001$; Fig. $8 \mathrm{c})$. The increased expression of Alpl by BMP-2 was not affected by the co-incubation of PTH.

\section{Discussion}

The role for TNAP in promoting ECM mineralisation has long been recognised and more recent studies have also identified PHOSPHO1 and nSMase 2 as important regulators of the biomineralisation process $[7,11,26]$. It is likely that nSMase2 and PHOSPHO1 function together to control the initiation of mineralisation via the liberation of $\mathrm{Pi}$ within the sheltered confines of osteoblast and chondrocyte MVs [1]. This possibility is supported by the observation that both PHOSPHO1 and nSMase2 are present in MV's [27]. In contrast, TNAP is bound, by means of a glycosylphosphatidylinositol anchor, to the outer leaflet of MV membranes, whereby its pyrophosphatase activity modulates the extravesicular Pi/PPi ratio so as to provide an environment conducive to mineral propagation $[1,11,13]$. The complete ablation of ECM mineralisation noted in Phosphol $^{-1-} ; \mathrm{Alpl}^{-1-}$ double-knockout mice underscores the importance and the non-redundant functions of these two phosphatases in regulating skeletal mineralisation [11]. Recent studies have further shown that MV's initiate mineralisation by a dual mechanism: PHOSPHO1-mediated intravesicular generation of $\mathrm{Pi}$ and phosphate transporter-mediated influx of Pi [28]. Despite knowledge of the function of these mineralisation-dependant proteins, there exists a hiatus in the knowledge surrounding their regulation. However, recent transcriptome sequencing analysis revealed that Phosphol is regulated by PTH in osteocytes [16]. This study was the impetus for this present investigation, where we sought to determine whether the catabolic effects of continuous PTH may be attributed to the regulation of PHOSPHO1 expression by 

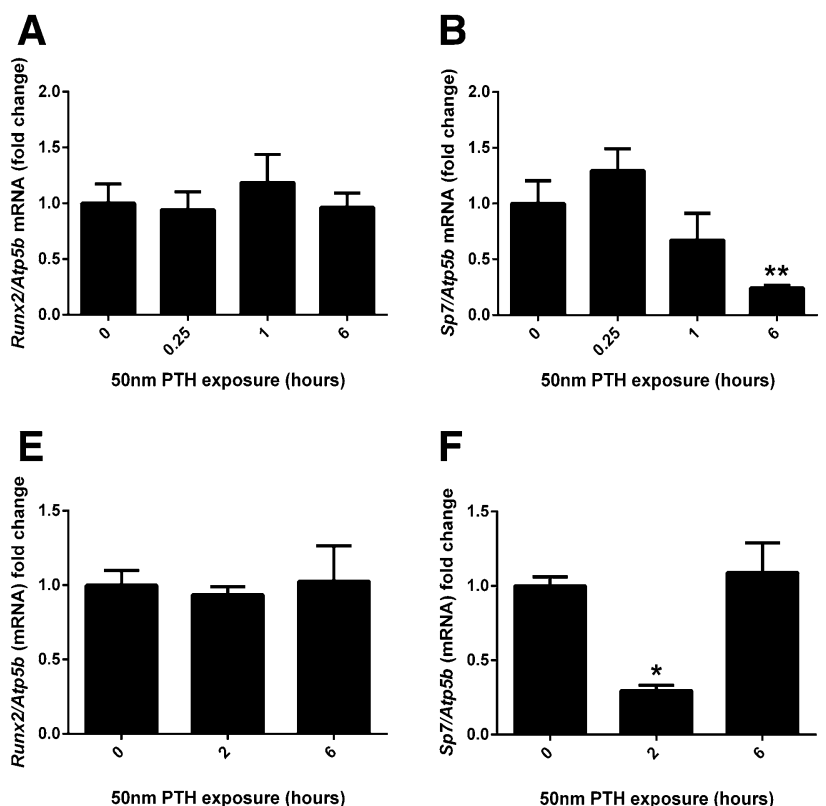

Fig. 7 Transcription factor expression in response to short-term PTH exposure. a Runx2, b $S p 7$, c Atf4 and d Trps 1 mRNA expression was assessed by RT-qPCR in MC3T3-C14 cell cultures in response to a short-term exposure to $50 \mathrm{nM}$ PTH on day 7 of culture. e Runx2,
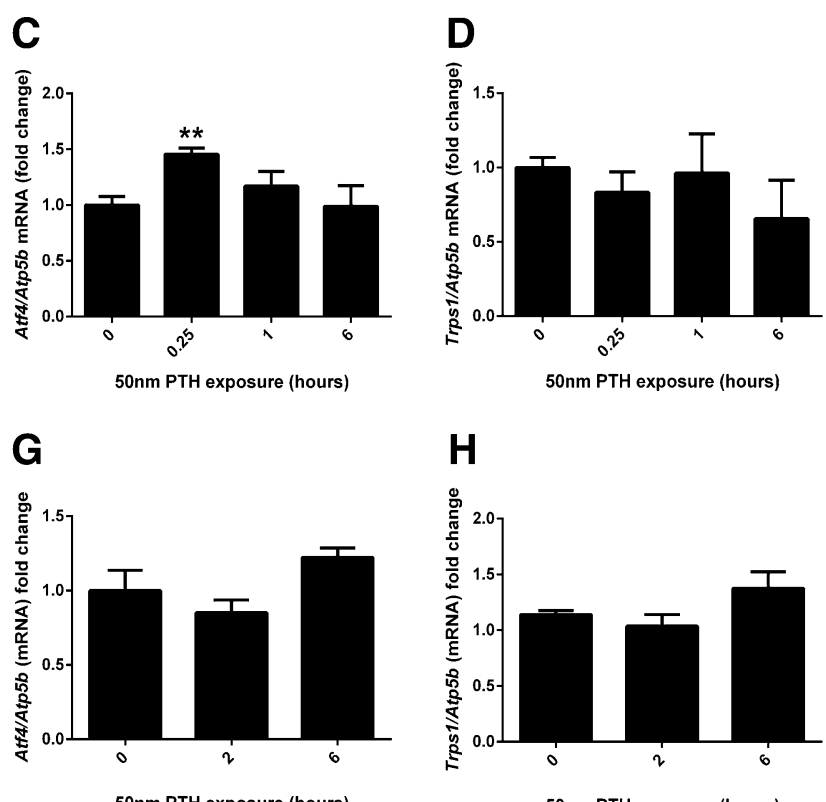

50nm PTH exposure (hours)

f $S p 7, \mathbf{g}$ Atf4 and $\mathbf{h} \operatorname{Trps} 1$ mRNA expression was assessed by RTqPCR in cultured murine calvariae in response to a short-term exposure to $50 \mathrm{nM}$ PTH. $N=3$; ${ }^{*} P<0.05 ; * * P<0.01$ in comparison with control-treated cultures

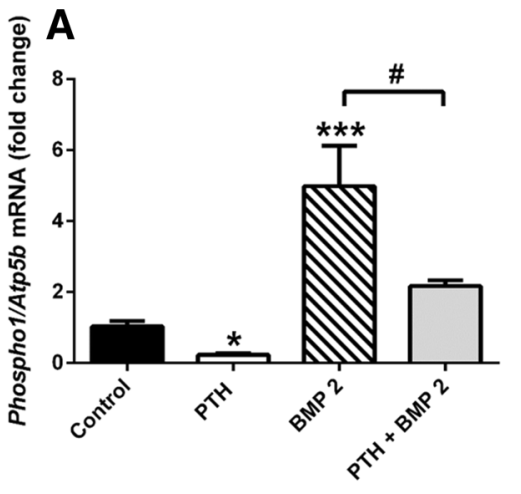

Fig. 8 Combined effects of continuous PTH and BMP-2 exposure on Phospho1, Alpl and Smpd3 expression in MC3T3-C14 cells. a Phosphol, b Alpl and c Smpd3 mRNA expression was assessed by RTqPCR in MC3T3-C14 cell cultures in response to a $24 \mathrm{~h}$ exposure to

osteoblasts, and also how this regulation may be coordinated with TNAP and nSMase2 expression.

This study has revealed that PHOSPHO1, nSMase2 and TNAP display coordinated and significant increases in the levels of transcript and protein prior to the onset of ECM mineralisation in the MC3T3-C14 osteoblast-like cell line. In particular, the similarities in the temporal rise of PHOSPHO1 and nSMase2 protein bolster the idea that these phosphatases work in tandem to generate Pi within MV during the initiation of mineralisation. Furthermore, PHOSPHO1 has recently been implicated in the biogenesis

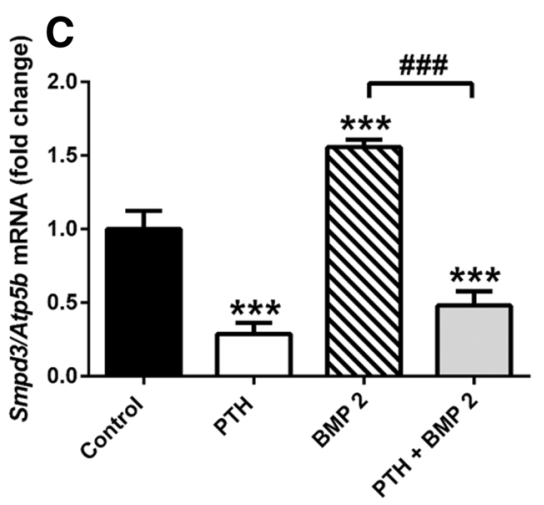

$50 \mathrm{nM}$ PTH, $300 \mathrm{ng} / \mathrm{mL}$ BMP-2 or both on day 6 of culture. $N=3$; $* P<0.05 ; * * * P<0.001$ in comparison to control treated cultures; ${ }^{\# \# \#} P<0.001$ in comparison with BMP-2-treated cultures

of MV [28], and as such its expression prior to the onset of mineralisation is crucial for the formation of MVs that ultimately contain a precipitated carbonate-substituted hydroxyapatitic mineral phase $[29,30]$. The higher expression of TNAP late on (day 10) noted in the time course of this study, highlights its role in the later stages of mineralisation where it allows the propagation of hydroxyapatite within the ECM beyond the confines of the MV membrane $[1,10]$.

This study provides the first evidence for the potent regulation of Phosphol and Smpd3 by PTH in osteoblast- 
like cells and cultured calvariae. Our data build on previous investigations which have sought to uncover the regulation of mineralisation-dependent genes by growth factors and hormones. The enhancement of nSMase 2 expression by BMP-2 signalling was recently reported in chondrocytes and $\mathrm{C} 2 \mathrm{C} 12$ myoblast cells [31, 32]. In this present study we have confirmed and expanded these findings, by revealing that continuous PTH exposure is able to significantly down-regulate Smpd3 expression even in the presence of BMP-2. Phosphol expression was similarly enhanced by BMP-2 exposure, with concomitant PTH exposure restoring expression levels to those of control cultures. The effects of BMP signalling on PHOSPHO1 regulation and the interaction between BMP and PTH signalling are intriguing and worthy of further investigation.

Whilst we found no interaction between PTH and BMP2 in the regulation of $A l p l$ expression, the effects of PTH alone on Alpl expression have been well documented. For example, intermittent exposure to PTH has been shown to stimulate ALP activity and bone nodule formation [33]. However, it should be noted that the inhibition of osteoblast differentiation by PTH has been reported in other studies $[34,35]$, and this variation in response may be explained by differences in osteoblast differentiation stage and PTH exposure time and dosage. PTH can exert entirely opposing effects on bone which depend upon the length of exposure. Intermittent exposure to PTH is anabolic to the skeleton, with reactivation of bone lining cells [36], inhibition of osteoblast apoptosis [37] and down-regulation of sclerostin, the potent negative regulator of Wnt signalling and bone formation [38]. Currently, rhPTH (1-34) (Teriparatide) is the only anabolic therapy for osteoporosis aimed at preventing both vertebral and non-vertebral fractures in post-menopausal women [39]. Contrastingly, continuous exposure to $\mathrm{PTH}$, as observed in hyperparathyroidism, is catabolic to bone, increasing osteoclastogenesis through upregulation of RANKL and inhibition of osteoprotegerin expression [22]. Knowledge of the effects of continuous exposure to PTH is, however, limited with regard to the regulation of mineralisation-promoting enzymes despite an overall increase in the remodelling rate observed in hyperparathyroidism [21]. The nature of the PTH administration described in this study, whereby PTH is added to the culture medium, and the medium is not changed before the experiments are stopped for collection of RNA/protein, which most closely resembles a continuous exposure to PTH. Indeed a number of investigations to date have demonstrated this method of PTH exposure to better model the catabolic effects of PTH [40, 41]. With bovine PTH (1-34) having a reported half-life of 10-12 h in vitro a biologically effective dose of $\sim 3 \mathrm{nM}$ should have remained in our cultures after $48 \mathrm{~h}$. However, as our time exposure studies show (Fig. 2d-e), Phosphol and Alpl gene expression levels were normalised by $48 \mathrm{~h}$. This suggests the lack of a biologically effective dose of PTH at this time point. In contrast, Smpd3 expression remained significantly down-regulated at $48 \mathrm{~h}$ possibly reflecting the increased sensitivity of this gene to PTH challenge (Fig. 2f). These data bring into question the levels of biologically active PTH remaining in our experimental cultures and highlight the limitations of this approach. However, this method of PTH exposure is in contrast to in vitro studies which seek to model the effects of intermittent PTH, whereby PTH is added to cell cultures for $1-6 \mathrm{~h}$ per $24-\mathrm{h}$ or 48 -h incubation cycle $[40,42]$. The findings presented here show for the first time that continuous administration of PTH to osteoblast cultures inhibits the expression of Phosphol and Smpd3.

The reduction of PHOSPHO1 and nSMase 2 may provide a novel mechanism by which continuous PTH exposure results in decreased bone mineral density. Indeed, continuous exposure to PTH reduced the extent of matrix mineralisation in MC3T3-C14 cell cultures (Fig. 1g) corroborating existing reports which assessed this protocol in rat calvarial osteoblast cultures [40]. Recent studies have provided data which highlight a role for PHOSPHO1 and nSMase 2 in the biogenesis of matrix vesicles [28, 43, 44]. It is possible, therefore, that the reduced mineralisation of MC3T3-C14 cell cultures observed in response to continuous exposure to PTH is due to both a decreased number of MVs being released and a decrease in their ability to initiate amorphous hydroxyapatite formation. Despite this, Alpl expression was induced in response to PTH in MC3T3-C14 cell cultures. An enhancement of Alpl expression and TNAP activity in response to a continuous exposure to PTH has previously been shown in periodontal ligament cells [45] and in the UMR-106 cell line [46], respectively. Even with this body of evidence in mind, the differential regulation of Phosphol and Alpl by PTH is perhaps surprising. The genetic ablation of Phosphol in the mouse leads to reduced serum TNAP activity and reduced Alpl expression in cultured chondrocytes derived from these mice [11]. Importantly, cultured hemi-calvariae exposed to continuous PTH exposure for $24 \mathrm{~h}$ displayed a down-regulation of $A l p l$ expression (Fig. 3b). The ex vivo culture of calvariae may provide a more physiologically relevant model of osteoblast behaviour due to the maintenance of both cell-cell and cell-matrix interactions in three-dimensional space.

To enact its effects, PTH binds to the PTH 1 receptor (PTH1R), a G-protein-coupled receptor, through a receptor binding domain between amino acids 15-24 [47]. Both PKA and PKC signal transduction pathways may be activated by binding of PTH to PTH1R. The effects of PTH are primarily elicited through the PKA pathway whereby, stimulatory $\mathrm{G} \alpha$ s proteins activate adenylate cyclase, with 
subsequent production of cAMP and phosphorylation of PKA [17]. A limited number of genes, for example those encoding the insulin-like growth-factor-binding protein 5 and transforming growth factor $\beta 1$, have been found to be regulated by $\mathrm{G} \alpha \mathrm{q}$ activation, which results in PKC activation, 1,4,5-inositol triphosphate production and a rise in intracellular $\mathrm{Ca}_{2}{ }^{+}$[25]. A critical role for the cAMP-PKA pathway in mediating the effects of PTH on PHOSPHO1, nSMase2 and TNAP expression in osteoblast cell cultures model has been revealed in this study. Firstly, the adenylyl cyclase agonist, forskolin, replicated the effects of PTH, whereas exposure to the PKC agonist, PMA, caused no effects. Furthermore, specific inhibition of the cAMP activation of PKA by the synthetic peptide PKI (5-24) abrogated the effects of PTH on Phospho1, Alpl and Smpd3 expression (Fig. 9). In support of a role for PKA signalling in the downregulation of the mineralisation-dependent enzymes, PHOSPHO1 and nSMase2, studies to date have shown the inhibition of both MC3T3-E1 matrix mineralisation and bone nodule formation by cultured rat calvarial cells with long-term exposure to forskolin [48, 49]. Our studies involving cycloheximide reveal that this genetic regulation is not dependent on protein synthesis. It is noted that cycloheximide appeared to partially affect the downregulation of Phosphol by PTH exposure (inducing a
$49.5 \%$ decrease as opposed to a $93.9 \%$ decrease by PTH alone). Despite this, it is well accepted that cycloheximide can exert off-target effects [50], and these may account for this difference. Finally, we assessed the expression of the osteogenic transcription factors Runx2, Sp7 and Atf4 and $\operatorname{Trps} 1$, which has recently been implicated in the regulation of Phosphol in a preodontoblastic cell line [51]. In both cultured murine calvariae and MC3T3-C14 cells, Sp7 expression was reduced in response to PTH. This suggests that osterix (encoded by the $S p 7$ gene), one of the master transcription factors regulating osteogenic differentiation, may be an important transcription factor in the regulation of both Phosphol and/or Smpd3 expression. The study of the transcriptional and epigenetic regulation of these mineralisation-dependent genes requires further study, and it is of interest to note that Runx 2 overexpression in mouse limb bud cells has been shown to result in elevated expression of both Phosphol and Smpd3 [52].

These data are novel for the PTH control of Phosphol and Smpd3 expression, but previous reports have demonstrated the involvement of the cAMP-PKA pathway in the regulation (both positive and negative) of TNAP activity in a variety of cell culture models of mineralisation $[33,40,45,46]$. The PTH-dependent enhancement of TNAP activity has been shown to be enacted through

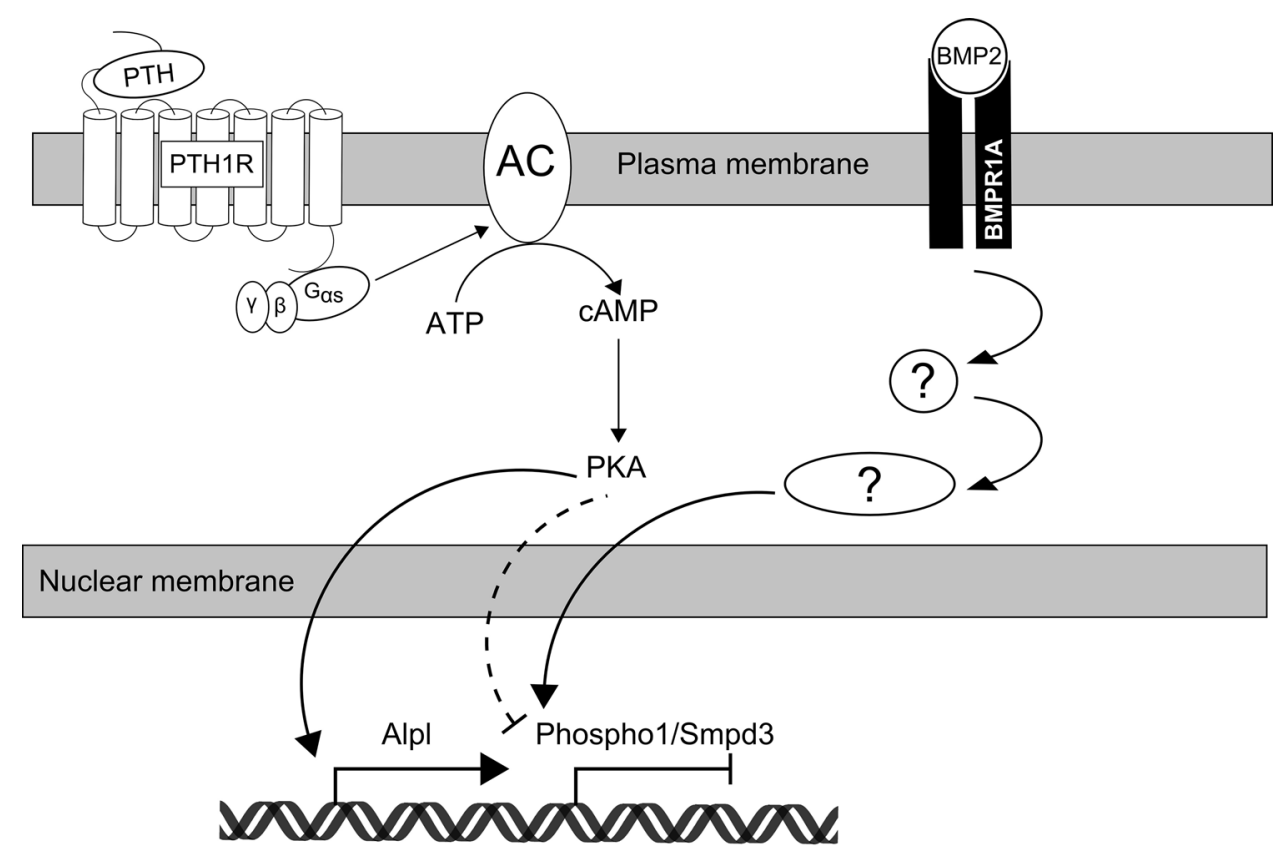

Fig. 9 Schematic representation of the signalling pathways mediating PTH and BMP-2 induced regulation of Phospho1, Smpd3 and Alpl in osteoblast cells. Recapitulation of the effects of PTH, through the use of the cAMP agonist, forskolin, and inhibition of the effects of PTH through the use of the PKA inhibitor, PKI (5-24) have implicated the cAMP/PKA pathway in mediating the down-regulation of Phosphol and Smpd3, and the enhancement of Alpl mRNA expression in response to PTH treatment. Initial studies have identified BMP-2 as an inducer of Phospho1, Smpd3 and Alpl expression, although the intracellular signalling pathway is yet to be elucidated. PTH, parathyroid hormone; PTH1R, parathyroid hormone receptor 1; AC, adenylyl cyclase; ATP, adenosine triphosphate; cAMP, cyclic adenosine monophosphate; PKA, protein kinase A; $\mathrm{BMP} 2$, bone morphogenic protein 2; BMPR1A, bone morphogenic protein receptor $1 \mathrm{~A}$ 
cAMP-PKA-mediated activation of p38 MAP kinase [53]. In the cultured hemi-cavariae experiments, cAMP stimulation by forskolin simulated the effects of PTH by inducing a downregulation of Phosphol, Alpl and Smpd3. In contrast to MC3T3-C14 cell cultures, however, PMA similarly induced the down-regulation of Alpl and Smpd3, and blockade of PKA activation by PKI (5-24) did not affect the PTH-induced downregulation of Smpd3. To date, Phosphol has been shown to be exclusively expressed by osteoblasts, hypertrophic chondrocytes, odontoblasts and calcifying vascular smooth muscle cells [8, 9, 54]. Smpd3 and $A l p l$, on the other hand, are more broadly expressed amongst different tissues and cell types. Although providing a more physiological environment, the presence of osteocytes, osteoclasts and stromal cells, as well as osteoblasts at differing stages of development in the ex vivo calvariae model, may be confounding our investigation of the signalling pathways in this model.

In summary, the data presented here provide evidence for of a temporal rise in the expression of key mineralisation effector enzymes, PHOSPHO1, TNAP and nSMase2, prior to the onset of in vitro mineralisation. This finding highlights the importance of these enzymes in this process. Furthermore, the potent negative regulation of Phosphol and Smpd3 by continuous exposure to PTH may provide an additional means of explaining the poor bone quality observed in response to sustained and elevated exposures to PTH.

\section{Materials and Methods}

\section{MC3T3-Clone 14 Cell Culture}

MC3T3 subclone 14 cells (MC3T3-C14) (ATCC, CRL$2594^{\mathrm{TM}}$ ) previously characterised as a highly mineralising clone of the MC3T3-E1 cell line $[13,55]$ were plated at $1 \times 104$ cells/cm 2 in six-well plates and cultured in maintenance medium ( $\alpha$-MEM containing $10 \%$ FBS and $0.5 \%$ Gentamicin) (Invitrogen, Paisley, UK) in a humidified atmosphere $\left(37^{\circ} \mathrm{C}, 5 \% \mathrm{CO} 2\right)$. Upon confluency, maintenance media was replaced with mineralisation media (maintenance medium supplemented with $50 \mu \mathrm{g} / \mathrm{ml}$ L-ascorbic acid and $1.5 \mathrm{mM}$ calcium chloride) (SigmaAldrich, Dorset, UK). Supplementation with calcium chloride successfully induces ECM mineralisation and negates the need for exogenous phosphate sources (e.g. beta-glycerophosphate) and therefore potential ectopic mineralisation or enhanced induction of Alpl expression $[13,56,57]$. Media was changed every 2-3 days for up to 10 days.

To examine the effects of continuous exposure of PTH on MC3T3-C14 gene and protein expression, the media was replaced with fresh mineralisation media containing either bPTH (1-34) (Sigma-Aldrich) (referred to as PTH) at various concentrations $(0.5-50 \mathrm{nM})$ or vehicle (PBS) and for various lengths of time (15 min-72 h). Unless otherwise stated, cells were cultured for 6 days (from confluency) under osteogenic conditions prior to the addition of PTH in order to avoid suppression of osteoblast differentiation [58, 59]. In experiments utilising BMP-2, cell cultures were treated with recombinant human BMP-2 (300 ng/mL; R \& D Systems, Abingdon, UK) for 24 h. To investigate the signalling pathways mediating the effects of PTH, cells were also treated with forskolin $(25 \mu \mathrm{M}$; SigmaAldrich), phorbol 12-myristate 13-acetate (PMA, $100 \mathrm{nM}$; Sigma-Aldrich), and PKI (5-24) (100 nM; Santa Cruz Biotechnology, Texas, USA) or appropriate vehicle controls for the time indicated. To assess the requirement of protein synthesis in the PTH regulation of Phosphol, Alpl and Smpd3, cells were pre-incubated for $2 \mathrm{~h}$ with cycloheximide (25 $\mu \mathrm{M}$; Sigma-Aldrich) or DMSO control prior to stimulation with either $50 \mathrm{nM}$ PTH or PBS for $6 \mathrm{~h}$.

\section{Murine Calvariae Culture}

Calvariae from 4- to 5-day-old C57Bl/6 J pups were dissected under sterile conditions. Hemi-calvariae were formed by cutting along the sagittal suture of the calvaria. Hemi-calvariae were cultured in 24-well plates in $350 \mu \mathrm{L}$ of calvaria culture medium ( $\alpha$-MEM containing $0.2 \% \mathrm{w} / \mathrm{v}$ bovine serum albumin, $0.05 \mathrm{mg} / \mathrm{ml}$ gentamicin, $1.25 \mu \mathrm{g} /$ $\mathrm{mL}$ Amphotericin B and $5 \mu \mathrm{g} / \mathrm{ml} \mathrm{L}$-ascorbic acid). After $24 \mathrm{~h}$ hemi-calvariae were treated with $50 \mathrm{nM}$ PTH, forskolin $(25 \mu \mathrm{M})$, PMA (100 nM) and PKI (5-24) (100 nM) for the times indicated.

\section{Quantification of Extracellular Matrix Mineralisation}

The effect of continuous PTH exposure on the ECM mineralisation of MC3T3-C14 cells was assessed by alizarin red staining and quantification. To examine the effects of continuous PTH on the ECM mineralisation, cell cultures were exposed to either $50 \mathrm{nM}$ PTH or PBS control for 10 days under osteogenic conditions; media changes, including fresh PTH or control, were performed every 48 h. On day 10 of culture, cells cultures were fixed in $4 \%$ paraformaldehyde for $5 \mathrm{~min}$ at room temperature. Cell monolayers were stained with aqueous $2 \%(\mathrm{w} / \mathrm{v})$ Alizarin red solution (Sigma-Aldrich) for $5 \mathrm{~min}$ at room temperature. The bound stain was solubilised in $10 \%$ cetylpyridinium chloride (Sigma-Aldrich) and the optical density of the resultant eluted solution measured by spectrophotometry at $570 \mathrm{~nm}$. 
Table 1 Primer sequences used to examine gene expression by RT-qPCR

\begin{tabular}{|c|c|c|c|}
\hline \multirow[t]{2}{*}{ Phosphol } & Forward primer & 5'-TTCTCATTTCGGATGCCA-3' & PrimerDesign, Southampton, U.K. \\
\hline & Reverse primer & 5'-TGAGGATGCGGCGGAAT-3' & \\
\hline \multirow[t]{2}{*}{ Alpl } & Forward primer & 5'-CTGCCACTGCCTACTTGTGT-3' & MWG Eurofins, Munich, Germany \\
\hline & Reverse primer & 5'-GATGGATGTGACCTCATTGC-3' & \\
\hline \multirow[t]{2}{*}{ Smpd3 } & Forward primer & 5'-ACACGACCCCCTTTCCTAATA-3' & MWG Eurofins, Munich, Germany \\
\hline & Reverse primer & 5'-GGCGCTTCTCATAGGTGGTG-3' & \\
\hline \multirow[t]{2}{*}{ Runx 2} & Forward primer & 5'-TGGCCGGGAATGATGAGAAC-3' & MWG Eurofins, Munich, Germany \\
\hline & Reverse primer & 5'-TGAAACTCTTGCCTCGTCCG-3' & \\
\hline \multirow[t]{2}{*}{$S p 7$} & Forward primer & Not disclosed by supplier & Qiagen, Crawley, U.K. \\
\hline & Reverse primer & Not disclosed by supplier & \\
\hline \multirow[t]{2}{*}{ Atf4 } & Forward primer & $5^{\prime}$-GTGGCCAAGCACTTGAAACC-3' & MWG Eurofins, Munich, Germany \\
\hline & Reverse primer & 5'-GGAAAAGGCATCCTCCTTGC-3' & \\
\hline \multirow[t]{2}{*}{ Trps1 } & Forward primer & 5'-ACAACGGCGAGCAGATTATTAG-3' & MWG Eurofins, Munich, Germany \\
\hline & Reverse primer & 5'-TAGTCAATGAACCCTGGGCTTCGTA-3' & \\
\hline
\end{tabular}

\section{Analysis of Gene Expression by Quantitative Real- Time PCR}

MC3T3-C14 cultures and hemi-calvariae were stopped at the desired time in culture and total RNA was extracted using the RNeasy mini kit and RNeasy mini lipid kit, respectively, (Qiagen, Crawley, UK) according the manufacturer's instructions. RNA concentration was assessed by absorbance at $260 \mathrm{~nm}$ and purity by A260/280 ratio using a NanoDrop spectrophotometer (Fisher Scientific, Loughborough, UK). Reverse transcription of RNA was carried out using Superscript II (Invitrogen) according to the manufacturer's instructions. Gene expression analysis, using the SYBR green detection method was performed on a Stratagene Mx3000P real-time qPCR system (Stratagene, California, USA). Samples were assessed in triplicate and normalised against the house keeping gene, Atp5b (Primer Design, Southampton, UK) as previously done in similar studies [13]. Relative gene expression was calculated using the $\Delta \Delta \mathrm{Ct}$ method [60] and expressed as a fold change compared to control. The primers used to amplify Phospho1, Smpd3, Alpl, Runx2, Sp7, Atf4 and Trps1 are shown in Table 1.

\section{Western Blotting}

Protein was extracted from cell monolayers using RIPA buffer (Invitrogen) with complete protease inhibitors (Roche, East-Sussex, UK). The concentration of the resultant protein lysates was determined by DC assay (BioRad, Hemel Hempstead, UK). Proteins $(10 \mu \mathrm{g})$ were separated on a $10 \%$ Bis-Tris gel and transferred to a nitrocellulose membrane. Membranes were blocked with Odyssey blocking buffer (LI-COR Biosciences, Nebraska,
USA) for $1 \mathrm{~h}$ at room temperature and probed overnight at $4{ }^{\circ} \mathrm{C}$ with anti-PHOSPHO1 (AbD Serotec, Martinsreid/ Planegg, Germany), anti-TNAP (R\&D, Abingdon, UK), anti-nSMase2 (Santa Cruz Biotechnology) and anti- $\beta$-actin (Cell Signalling Technology, Hitchin, UK) antibodies diluted 1:1000, 1:500, 1:500 and 1:1000 respectively in Odyssey blocking buffer with $1.875 \%$ Tween 20 . After washing in PBS, membranes were probed with the appropriate secondary antibodies for $50 \mathrm{~min}$ at room temperature (1:1250 dilution in Odyssey blocking buffer). The resulting blots were subsequently washed in PBS and visualised using the LI-COR Odyssey infrared scanner and software (LI-COR biosciences) with a scan resolution of $169 \mu \mathrm{m}$. Quantification of the fluorescence of each protein of interest was performed using Image Studio Lite version 5.0 (LI-COR biosciences) and normalised against beta-actin. The resulting quantification was expressed as fold change compared with control-treated samples.

\section{Statistical Analysis}

Data analysis was performed using GraphPad Prism 6 (GraphPad Prism Inc., California, USA), and data are presented as mean \pm S.E.M. Comparisons between datasets were carried out using ANOVA or two-way ANOVA with post hoc Tukey's tests where appropriate. $P<0.05$ was considered to be significant and noted as '*'; $P$ values of $<0.01$ and $<0.001$ were noted as ' $* *$ ' and ' $* * *$,' respectively.

Acknowledgments The authors wish to acknowledge Miss Elaine Seawright (The Roslin Institute, University of Edinburgh) for her technical assistance. We are also grateful to the Biotechnology and Biological Sciences Research Council (BBSRC) for Institute Strategic Programme Grant Funding (BB/J004316/1) and for Doctoral Training 
Partnership funding to D.A.H. (BB/J01446X/1). We would also like to acknowledge Arthritis Research UK for support to K.A.S. (Grant Number-20413).

\section{Compliance with Ethical Standards}

Conflict of interest D. A. Houston, K. Myers, V. E. MacRae, K. A. Staines and C. Farquharson declare that they have no conflict of interest.

Human and Animal Rights and Informed Consent All procedures complied with the UK Animals (Scientific Procedures) Act 1986 and were reviewed and approved by the ethics committee of The Roslin Institute, University of Edinburgh.

Open Access This article is distributed under the terms of the Creative Commons Attribution 4.0 International License (http://creative commons.org/licenses/by/4.0/), which permits unrestricted use, distribution, and reproduction in any medium, provided you give appropriate credit to the original author(s) and the source, provide a link to the Creative Commons license, and indicate if changes were made.

\section{References}

1. Millán JL (2013) The role of phosphatases in the initiation of skeletal mineralization. Calcif Tissue Int 93(4):299-306

2. Fedde KN, Blair L, Silverstein J, Coburn SP, Ryan LM, Weinstein RS et al (1999) Alkaline phosphatase knock-out mice recapitulate the metabolic and skeletal defects of infantile hypophosphatasia. J Bone Miner Res 14(12):2015-2026

3. Anderson HC, Hsu HH, Morris DC, Fedde KN, Whyte MP (1997) Matrix vesicles in osteomalacic hypophosphatasia bone contain apatite-like mineral crystals. Am J Pathol 151(6): $1555-1561$

4. Anderson HC, Sipe JB, Hessle L, Dhamyamraju R, Atti E, Camacho NP et al (2004) Impaired calcification around matrix vesicles of growth plate and bone in alkaline phosphatase-deficient mice. Am J Pathol 164(3):841-847

5. Roberts SJ, Stewart AJ, Sadler PJ, Farquharson C (2004) Human PHOSPH01 exhibits high specific phosphoethanolamine and phosphocholine phosphatase activities. Biochem J 382:59-65

6. Stewart AJ, Roberts SJ, Seawright E, Davey MG, Fleming RH, Farquharson C (2006) The presence of PHOSPHO1 in matrix vesicles and its developmental expression prior to skeletal mineralization. Bone 39(5):1000-1007

7. Roberts S, Narisawa S, Harmey D, Millan JL, Farquharson C (2007) Functional involvement of PHOSPHO1 in matrix vesiclemediated skeletal mineralization. J Bone Miner Res 22(4):617627

8. Houston B, Stewart AJ, Farquharson C (2004) PHOSPHO1-a novel phosphatase specifically expressed at sites of mineralisation in bone and cartilage. Bone 34(4):629-637

9. McKee MD, Yadav MC, Foster BL, Somerman MJ, Farquharson C, Millán JL (2013) Compounded PHOSPHO1/ALPL deficiencies reduce dentin mineralization. J Dent Res 92(8):721-727

10. Huesa C, Yadav MC, Finnila MAJ, Goodyear SR, Robins SP, Tanner KE et al (2011) PHOSPHO1 is essential for mechanically competent mineralization and the avoidance of spontaneous fractures. Bone 48(5):1066-1074

11. Yadav MC, Simao AMS, Narisawa S, Huesa C, McKee MD, Farquharson $\mathrm{C}$ et al (2011) Loss of skeletal mineralization by the simultaneous ablation of PHOSPHO1 and alkaline phosphatase function: a unified model of the mechanisms of initiation of skeletal calcification. J Bone Miner Res 26(2):286-297
12. Rodriguez-Florez N, Garcia-Tunon E, Mukadam Q, Saiz E, Oldknow KJ, Farquharson C et al (2015) An investigation of the mineral in ductile and brittle cortical mouse bone. J Bone Miner Res 30(5):786-795

13. Huesa C, Houston D, Kiffer-Moreira T, Yadav MC, Luis Millan J, Farquharson C (2015) The functional co-operativity of tissuenonspecific alkaline phosphatase (TNAP) and PHOSPHO1 during initiation of skeletal mineralization. Biochem Biophys Rep 4:196-201

14. Khavandgar Z, Poirier C, Clarke CJ, Li JJ, Wang N, McKee MD et al (2011) A cell-autonomous requirement for neutral sphingomyelinase 2 in bone mineralization. J Cell Biol 194(2):277289

15. Khavandgar Z, Alebrahim S, Eimar H, Tamimi F, McKee MD, Murshed M (2013) Local regulation of tooth mineralization by sphingomyelin phosphodiesterase 3. J Dent Res 92(4):358-364

16. John HCS, Meyer MB, Benkusky NA, Carlson AH, Prideaux M, Bonewald LF et al (2015) The parathyroid hormone-regulated transcriptome in osteocytes: parallel actions with 1,25-dihydroxyvitamin D-3 to oppose gene expression changes during differentiation and to promote mature cell function. Bone 72 : $81-91$

17. Silva BC, Bilezikian JP (2015) Parathyroid hormone: anabolic and catabolic actions on the skeleton. Curr Opin Pharmacol 22:41-50

18. Qin L, Qiu P, Wang LQ, Li X, Swarthout JT, Soteropoulos P et al (2003) Gene expression profiles and transcription factors involved in parathyroid hormone signaling in osteoblasts revealed by microarray and bioinformatics. J Biol Chem 278(22):1972319731

19. Krishnan V, Moore TL, Ma YFL, Helvering LM, Frolik CA, Valasek KM et al (2003) Parathyroid hormone bone anabolic action requires Bbfa1/Runx2-dependent signaling. Mol Endocrinol 17(3):423-435

20. Locklin RM, Khosla S, Turner RT, Riggs BL (2003) Mediators of the biphasic responses of bone to intermittent and continuously administered parathyroid hormone. J Cell Biochem 89(1):180190

21. Eriksen EF (2002) Primary hyperparathyroidism: lessons from bone histomorphometry. J Bone Miner Res 17:N95-N97

22. Ma YFL, Cain RL, Halladay DL, Yang XH, Zeng QQ, Miles RR et al (2001) Catabolic effects of continuous human PTH (1-38) in vivo is associated with sustained stimulation of RANKL and inhibition of osteoprotegerin and gene-associated bone formation. Endocrinology 142(9):4047-4054

23. Dempster DW, Parisien M, Silverberg SJ, Liang XG, Schnitzer M, Shen V et al (1999) On the mechanism of cancellous bone preservation in postmenopausal women with mild primary hyperparathyroidism. J Clin Endocrinol Metab 84(5):1562-1566

24. Iida-Klein A, Lu SS, Kapadia R, Burkhart M, Moreno A, Dempster DW et al (2005) Short-term continuous infusion of human parathyroid hormone 1-34 fragment is catabolic with decreased trabecular connectivity density accompanied by hypercalcemia in C57BL/J6 mice. J Endocrinol 186(3):549-557

25. Qin L, Raggatt LJ, Partridge NC (2004) Parathyroid hormone: a double-edged sword for bone metabolism. Trends Endocrinol Metab 15(2):60-65

26. Robison R (1923) The possible significance of hexosephosphoric esters in ossification. Biochem J 17(2):286-293

27. Mebarek S, Abousalham A, Magne D, Le Duy D, BandorowiczPikula J, Pikula S et al (2013) Phospholipases of mineralization competent cells and matrix vesicles: roles in physiological and pathological mineralizations. Int J Mol Sci 14(3):5036-5129

28. Yadav MC, Bottini M, Cory E, Bhattacharya K, Kuss P, Narisawa $S$ et al (2016) Skeletal mineralization deficits and impaired biogenesis and function of chondrocyte-derived matrix vesicles in 
phospho1 $1^{-/-}$and phospho1/Pit1 double knockout mice. J Bone Miner Res 31(6): 1275-1286

29. Anderson HC (1969) Vesicles associated with calcification in matrix of epiphyseal cartilage. J Cell Biol 41(1):59-72

30. Register TC, McLean FM, Low MG, Wuthier RE (1986) Roles of alkaline-phosphatase and labile internal mineral in matrix vesicle-mediated calcification. Effect of selective release of membrane-bound alkaline-phosphatase and treatment with isosmotic PH-6 buffer. J Biol Chem 261(20):9354-9360

31. Kakoi H, Maeda S, Shinohara N, Matsuyama K, Imamura K, Kawamura I et al (2014) Bone morphogenic protein (BMP) signaling up-regulates neutral sphingomyelinase 2 to suppress chondrocyte maturation via the Akt protein signaling pathway as a negative feedback mechanism. J Biol Chem 289(12):8135-8150

32. Chae Y-M, Heo S-H, Kim J-Y, Lee J-M, Ryoo H-M, Cho J-Y (2009) Upregulation of smpd3 via BMP2 stimulation and Runx2. BMB Rep 42(2):86-90

33. Terakado A, Tagawa M, Goto S, Yamazaki M, Moriya H, Fujimura S (1995) Elevation of alkaline phosphatase activity induced by parathyroid hormone in osteoblast-like cells from the spinal hyperostotic mouse TWY (twy/twy). Calcif Tissue Int 56(2):135139

34. Zerega B, Cermelli S, Bianco P, Cancedda R, Cancedda FD (1999) Parathyroid hormone $\mathrm{PTH}(1-34)$ and parathyroid hormone-related protein PTHrP(1-34) promote reversion of hypertrophic chondrocytes to a prehypertrophic proliferating phenotype and prevent terminal differentiation of osteoblast-like cells. J Bone Miner Res 14(8):1281-1289

35. van der Horst G, Farih-Sips H, Lowik C, Karperien M (2005) Multiple mechanisms are involved in inhibition of osteoblast differentiation by PTHrP and PTH in KS483 cells. J Bone Miner Res 20(12):2233-2244

36. Dobnig H, Turner RT (1995) Evidence that intermittent treatment with parathyroid-hormone increases bone-formation in adult-rats by activation of bone lining cells. Endocrinology 136(8):36323638

37. Jilka RL, Weinstein RS, Bellido T, Roberson P, Parfitt AM, Manolagas SC (1999) Increased bone formation by prevention of osteoblast apoptosis with parathyroid hormone. J Clin Invest. 104(4):439-446

38. Kramer I, Keller H, Leupin O, Kneissel M (2010) Does osteocytic SOST suppression mediate PTH bone anabolism? Trends Endocrinol Metab 21(4):237-244

39. Poole KES, Reeve J (2005) Parathyroid hormone-a bone anabolic and catabolic agent. Curr Opin Pharmacol 5(6):612-617

40. Ishizuya T, Yokose S, Hori M, Noda T, Suda T, Yoshiki S et al (1997) Parathyroid hormone exerts disparate effects on osteoblast differentiation depending on exposure time in rat osteoblastic cells. J Clin Invest 99(12):2961-2970

41. Liu Q, Wan Q, Yang R, Zhou H, Li Z (2012) Effects of intermittent versus continuous parathyroid hormone administration on condylar chondrocyte proliferation and differentiation. Biochem Biophys Res Commun 424(1):182-188

42. Lotinun S, Sibonga JD, Turner RT (2002) Differential effects of intermittent and continuous administration of parathyroid hormone on bone histomorphometry and gene expression. Endocrine 17(1):29-36

43. Kapustin AN, Chatrou MLL, Drozdov I, Zheng Y, Davidson SM, Soong D et al (2015) Vascular smooth muscle cell calcification is mediated by regulated exosome secretion. Circ Res 116(8):13121323

44. Thouverey C, Malinowska A, Balcerzak M, Strzelecka-Kiliszek A, Buchet R, Dadlez M et al (2011) Proteomic characterization of biogenesis and functions of matrix vesicles released from mineralizing human osteoblast-like cells. J Proteom 74(7):1123-1134
45. Wolf M, Jaeger A, Abuduwali N, Goetz W, Lossdoerfer S (2013) Continuous PTH modulates alkaline phosphatase activity in human PDL cells via protein kinase $\mathrm{C}$ dependent pathways in vitro. Ann Anat Anat Anz 195(5):455-460

46. Kano J, Sugimoto T, Fukase M, Chihara K (1994) Direct involvement of cAMP-dependent protein kinase in the regulation of alkaline phosphatase activity by parathyroid hormone (PTH) and PTH-Related peptide in osteoblastic UMR-106 cells. Biochem Biophys Res Commun. 199(1):271-276

47. Mannstadt M, Juppner H, Gardella TJ (1999) Receptors for PTH and PTHrP: their biological importance and functional properties. Am J Physiol Renal Physiol 277(5):F665-F675

48. Kaneki H, Takasugi I, Fujieda M, Kiriu M, Mizuochi S, Ide H (1999) Prostaglandin E-2 stimulates the formation of mineralized bone nodules by a cAMP-independent mechanism in the culture of adult rat calvarial osteoblasts. J Cell Biochem 73(1):36-48

49. Tintut Y, Parhami F, Le V, Karsenty G, Demer LL (1999) Inhibition of osteoblast-specific transcription factor $\mathrm{Cbfa} 1$ by the cAMP pathway in osteoblastic cells: ubiquitin/proteosome-dependent regulation. J Biol Chem 274(41):28875-28879

50. McMahon D (1975) Cycloheximide is not a specific inhibitor of protein-synthesis in vivo. Plant Physiol 55(5):815-821

51. Kuzynski M, Goss M, Bottini M, Yadav MC, Mobley C, Winters $\mathrm{T}$ et al (2014) Dual role of the Trps 1 transcription factor in dentin mineralization. J Biol Chem 289(40):27481-27493

52. Nishimura R, Wakabayashi M, Hata K, Matsubara T, Honma S, Wakisaka $S$ et al (2012) Osterix regulates calcification and degradation of chondrogenic matrices through matrix metalloproteinase 13 (MMP13) expression in association with transcription factor Runx2 during endochondral ossification. J Biol Chem 287(40):33179-33190

53. Rey A, Manen D, Rizzoli R, Ferrari SL, Caverzasio J (2007) Evidences for a role of p38 MAP kinase in the stimulation of alkaline phosphatase and matrix mineralization induced by parathyroid hormone in osteoblastic cells. Bone 41(1):59-67

54. Kiffer-Moreira T, Yadav MC, Zhu D, Narisawa S, Sheen C, Stec B et al (2013) Pharmacological inhibition of PHOSPHO1 suppresses vascular smooth muscle cell calcification. J Bone Miner Res 28(1):81-91

55. Wang D, Christensen K, Chawla K, Xiao GZ, Krebsbach PH, Franceschi RT (1999) Isolation and characterization of MC3T3E1 preosteoblast subclones with distinct in vitro and in vivo differentiation mineralization potential. J Bone Miner Res 14(6):893-903

56. Javaheri B, Carriero A, Staines KA, Chang YM, Houston DA, Oldknow KJ et al (2015) Phospho1 deficiency transiently modifies bone architecture yet produces consistent modification in osteocyte differentiation and vascular porosity with ageing. Bone 81:277-291

57. Chang MK, Raggatt L-J, Alexander KA, Kuliwaba JS, Fazzalari NL, Schroder K et al (2008) Osteal tissue macrophages are intercalated throughout human and mouse bone lining tissues and regulate osteoblast function in vitro and in vivo. J Immunol 181(2):1232-1244

58. Bellows CG, Ishida H, Aubin JE, Heersche JNM (1990) Parathyroid hormone reversibly suppresses the differentiation of osteoprogenitor cells into functional osteoblasts. Endocrinology 127(6):3111-3116

59. Isogai $Y$, Akatsu $T$, Ishizuya $T$, Yamaguchi A, Hori M, Takahashi $\mathrm{N}$ et al (1996) Parathyroid hormone regulates osteoblast differentiation positively or negatively depending on the differentiation stages. J Bone Miner Res 11(10):1384-1393

60. Livak KJ, Schmittgen TD (2001) Analysis of relative gene expression data using real-time quantitative PCR and the 2(T)(Delta Delta C) method. Methods 25(4):402-408 